\title{
Das Durkheim-Simmelsche Projekt einer ,rein wissenschaftlichen Soziologie“ im Schatten der Dreyfus-Affäre
}

\section{Otthein Rammstedt}

Universität Bielefeld, Fakultät für Soziologie, PF 100131, D-33501 Bielefeld

Zusammenfassung: Der Beitrag wirft ein neues Licht auf die Beziehung Durkheim/Simmel. Die Gründung der Année sociologique war zugleich ein Versuch beider, eine neue Soziologie zu initiieren. In der Dreyfus-Affäre fühlte sich Durkheim aber gezwungen, die Zusammenarbeit mit Simmel aufzukündigen. Das hieß jedoch nicht, den Dialog abzubrechen, wie bisher fur 1898 angenommen wird, dieser setzte sich vielmehr in ihren soziologischen Schriften fort. Der Beitrag rekonstruiert diese Kontinuität in Hinblick auf Theoriekonstruktion und insbesondere auf Religionssoziologie.

In den Auseinandersetzungen um die Soziologie im letzten Dezennium des 19. Jahrhunderts gewannen die jungen Georg Simmel und Emile Durkheim schnell an Profil, ja, wurden zu den Protagonisten einer wissenschaftlichen Soziologie. „M. Simmel et M. Durkheim semblent caractérisés par un vif souci de mettre en lumière ce qu'il y a de proprement social dans le complexus des phénomènes historiques, et de spécifier le point de vue particulier à la sociologie“", stellte Célestin Bouglé damals fest; und dieses präzisierend fuhr er fort: „Pour eux, il semble que la tâche propre du sociologue ne soit pas de faire une sorte de synthèse des sciences particulières, mais de démêler, dans tous les ordres de phénomènes historiques, économiques ou politiques, l'influence spécifique de la société, c'est-à-dire le résultat des rapports qui unissent les individus, et l'action de tous sur chacun" (Bouglé 1898: 123). Daß Bouglé das so prononciert im Eröffnungsband der „Année sociologique" betonte und daß die skizzierte Soziologieumschreibung der Programmatik im Préface zu diesem Band entsprach, ist nicht zufällig; denn diesem soziologischen Programm fühlten sich Bouglé wie auch Durkheim und Simmel verpflichtet.

Anläßlich des 100-jährigen Jubiläums der „Année sociologique" auf das Verhältnis von Emile Durkheim und Georg Simmels in Hinblick auf deren Konstitution zu sprechen zu kommen, scheint angemessen, da dieser Aspekt bisher nur randständig behandelt und als Intermezzo gedeutet wurde (vgl. Besnard 1979; Lukes 1973: 289-296). Anlaß, dieses Verhältnis gerade jetzt anzusprechen, ist zudem, daß sich 1998 der 140. Geburtstag von Durkheim und Simmel jährt.
Mit der „Année sociologique“ beabsichtigte Durkheim, ein Publikationsorgan für einen rein wissenschaftlichen Zugang zur Soziologie schaffen; damit wollte er sich bewußt in Distanz zu René Worms' "Revue internationale de sociologie" bringen; und er wußte zudem, daß die ihm (wissenschafts-)politisch nahestehende „Revue de Métaphysique et de Morale" von ihrem Philosophiebegriff her Soziologie eigentlich auszuklammern gedachte und daher bereitwillig auf den Titel ihrer Rubrik „l'Année sociologique“ zu seinen Gunsten zu verzichten bereit war; und er mußte auch feststellen, daß die Soziologie in anderen Ländern sich scheinbar schneller konstituierte als in Frankreich - wofür ihm die Zeitschriftengründungen standen: das „American Journal of Sociology“" (gegr. 1895), die „Zeitschrift für Socialforschung“ (gegr. 1896) oder die "Rivista italienna di Sociologia“" (gegr. 1897).

Aber im Gegensatz zu Ward, Wolff und vor allem Worms hatte Durkheim kein Organ für alle Spielarten der Soziologie, sondern nur für ,une sociologie vraiment scientifique" im Blick. ${ }^{2}$ Und wenn er in diesem Zusammenhang Simmel anging, sich bei der Gründung zu beteiligen, so, da er in ihm einen Gleichgesinnten sah. Durkheim wußte von Simmels sozialwissenschaftlichen, insbesondere soziologischen Publikationen, spätestens durch Bouglé; durch ihn hatte Durkheim auch erfahren,

\footnotetext{
1 Vgl. Brief Emile Durkheims an Célestin Bouglé vom 20. Juni 1897, in EDT 2:397-399. - Auch Simmel hatte bereits 1892 die Gründung einer soziologischen Zeitschrift ins Auge gefaßt, vgl. Brief Georg Simmels an Lester F. Ward vom 24.2. 1893, in GSG 22/23.

${ }^{2}$ Bouglé (1895: 147) betont ausdrücklich, daB Durkheim in der Absicht, eine solche zu konstituieren, sich mit Simmel einer Meinung wisse.
} 
daß Simmel mit dem „Problem der Sociologie“ von 1894 sein Programm umrissen hatte, das er engagiert verbreitete und für das er sich so einzusetzen vorhatte, da $B$ er andere wissenschaftliche Interessen hintanstellte. Und Durkheim konstatierte, daß Simmel unabhängig von ihm aber gleich ihm eine rein wissenschaftliche Soziologie anstrebte.

Simmel ließ sich zur Mitarbeit an der „Année sociologique" lange bitten, sagte dann aber zu, neben Durkheim den anderen grundsätzlichen Beitrag zum Eröffnungsheft zu schreiben.

Mit „Selbsterhaltung der Gesellschaft", wie der ursprüngliche Titel des Beitrages hieß, griff Simmel einen zentralen Aspekt auf, der die Absetzung zu Spencer - und damit zu der alten und den gegenwärtig noch vertretenen evolutionär organizistisch orientierten Soziologen (Schäffle, Espinas, Worms) - dokumentierte, ohne Darwin damit auszuhebeln. Aber wenn Simmel seinen Beitrag „Selbsterhaltung der Gesellschaft" nur - kokett untertreibend - als Exemplifikation des methodischen Prinzips ausgab, „das meiner Ansicht nach allein eine Sociologie als selbstständige Wissenschaft begründen kann: die Abstraktion der Vergesellschaftung als Form und Art des Mit- und Füreinander-Seins von den konkreten Zuständen, Interessen, Empfindungen, welche den Inhalt jener Form bilden" (GSG 5: 371; entsprechend Simmel 1898b: 108), so sprach er primär die Ebene an, die er als gemeinsame mit Durkheim annahm. Und Durkheim nutzte vice versa die sich ihm damit gebotene Chance, indem er den scheinbar vereinnahmenden Anspruch des Titels des Simmel'schen Beitrags durch die Änderung in „Comment les formes sociales se maintiennent" behob und damit zugleich erstmals auf den soziologisch individuellen Ansatz Simmels hinwies.

Simmel hatte seinen Beitrag „Selbsterhaltung der Gesellschaft" als programmatisch für die „Année sociologique" gehalten, da sie methodisch fordere, „das Gesamtgebiet der Geschichte nach den Gesetzlichkeiten und Entwickelungen der Gesellschaft als solcher zu untersuchen" (1898a). Und in diesem Sinne, sich als Mitstreiter Durkheims wähnend, schrieb er unmittelbar nach Fertigstellung dieses Beitrages, nämlich ab August 1897, seine „Soziologie der Religion“, eine Studie, die im Februar 1898 in der „Neuen Deutschen Rundschau“ erschien.

Mag aus heutiger Sicht Simmels „Soziologie der Religion" am Anfang der Religionssoziologie stehen, so lag nicht die Thematisierung der Religion in Simmels Absicht, sondern ihre soziologische Behandlung. Denn neben der besonderen Beachtung der Religion in den evolutionstheoretisch orientierten Sozialwissenschaften, besonders in der Ethnologie und Anthropologie, drängten sich Religion und Kirche als aktuelles Thema in Frankreich wie in Deutschland auf; war es für Durkheim in Frankreich besonders die Dreyfus-Affäre, in der die katholische Kirche politisch Stellung bezog, was sich in der Diskussion um die 1400-Jahr-Feier der Clodwig-Taufe 1897 wiederholte, so wurde es für Simmel in Deutschland faßbar in der Diskussion um die „Ethische Kultur", die als Amerika-Import mit Verlust der Kirchen- bzw. Sektenbindung als sozial-ethische Bewegung auf große Resonanz stieß. ${ }^{3}$ So wundert es nicht, daß Durkheim bereits 1894/95 in seinem Soziologiekurs Religion thematisierte und daß Simmel ab 1897 Religionsphilosophie lehrte, auch im November 1897 vor dem „Sozialwissenschaftlichen Studentenverein" eine "Soziologie des religiösen Wesens" vortrug und Mitarbeiter der Zeitschrift „Ethische Kultur" wurde. ${ }^{4}$ Vor diesem Hintergrund ist dann der Anlaß für Simmels "Soziologie der Religion" im Programm der "Année sociologique" zu suchen, also in der vermeintlichen mit Durkheim gemeinsamen Verpflichtung für die Konstitution einer wissenschaftlichen Soziologie. So gab sich der Aufsatz als Beitrag zur Soziologie, und er sprach mit Religion einen Bereich an, der in der „Préface" neben Recht, Kultur, Moralstatistik und Ökonomie genannt wird, deren Geschichte „c'est là que ce trouvent les matériaux avec lesquels la sociologie se doit construire" (AS 1: 1). Und es war für Simmel daher auch nicht Anliegen, Religion als „soziale Objektivation" im Sinne von Lazarus darzulegen, auch nicht Religion intern als soziologisch zu entfalten - so wies er schon im zweiten Band seiner „Einleitung in die Moralwissenschaft" (1893) darauf hin, daß „die Hypostasierung der sozialen Kräfte in Gott... der Höhepunkt des soziologischen Begriffsrealismus" sei (GSG 4: 127), und entsprechend umschrieb er in „Zur Soziologie der Religion" dann Religion als „Substanzialisierung dessen..., was als Form und Funktion das Gruppenleben reguliert"(GSG 5:280) - ja, er betont in diesem Aufsatz einleitend, ganz im Sinne der „Pré-

${ }^{3}$ Gregor von Gizycki, der als a.o.Professor der Philosophie an derselben Fakultăt lehrte wie Simmel, stand der "Ethischen Kultur" in Deutschland vor, für die sich auch Ignaz Jastrow, Harald Höffding wie Gertrud Kantorowicz 1897 stark engagierten. - Vgl. Köhnke 1996: 284 ff.

4 "Zur Psychologie und Soziologie der Lüge" wie „Ueber Geiz, Verschwendung und Armut" erschienen dort 1899. 
face“ von „Année sociologique“, daß man den Ursprung und die Geschichte der Religion nur analysieren könne, wenn man ,alle Impulse, Ideen, Verhältnisse, die auf dem Gebiet wirksam werden, inventarisiert" (GSG 5: 266); Simmel wollte an Religion das ihm Neue exemplifizieren, das methodisch mit der analytischen Trennung von Form und Inhalt zu suchen sei.

$* * *$

Simmels „Zur Soziologie der Religion“ erschien Anfang 1898, wenige Tage nach dem Eröffnungsband der „Année sociologique“, zu einem Zeitpunkt, an dem Simmel die Distanzierungsbemühungen Durkheims erstmals greifen konnte. ${ }^{5}$

Als Simmel die "Année sociologique" in Händen hielt, stellte er fest, daß sein Beitrag „Comment les formes sociales se maintiennent" ohne Rücksprache noch einmal willkürlich gekürzt und in der Übersetzung von Durkheim sprachlich geändert worden war. Er mußte Durkheim daraufhin unmittelbar seine Empörung mitgeteilt haben; dabei waren es zuerst einmal die Umgangsformen, die Simmel brüskierten und die ihn veranlaßten, die Mitarbeit an der „Année sociologique" zu überdenken. ${ }^{6}$ Aber es war mehr als nur die Verletzung von Umgangsformen. Und so kam Durkheim Simmels Reaktion entgegen - er sah ab sofort die Zusammenarbeit beendet. Denn Durkheim war beim Übersetzen des Simmel-Textes mehrmals auf Passagen gestoßen, die ihn zweifeln ließen, ob die von ihm ausgesprochene Einladung an Simmel richtig gewesen war: einige Passagen Simmels ließen sich nach Durkheim nur so deuten, daß sie verdeckte Angriffe auf die Dreyfusards enthielten und dies wurde scheinbar durch eine offen erkennbare $\mathbf{A b}$ sage an den Zionismus verstärkt, was Durkheim so überraschte, daß er bei Bouglé brieflich nachfragte, ob Simmel wirklich Jude sei. Unter diesen von ihm gesehenen politischen und weltanschaulichen Gegensätzen war ihm eine Trennung nur recht.

Als Durkheim daher nun Simmels ,Zur Soziologie der Religion“ las, ging es ihm nicht mehr wie Sim-

s Leider muß die Korrespondenz zwischen Durkheim und Simmel als verloren gelten, so daß der Hergang des Konflikts nur spekulativ erfaßt werden kann.

${ }^{6}$ Da Simmel nach den schlechten Erfahrungen mit der Übersetzung seiner Arbeit für Worms Bouglé - und wohl auch Durkheim - davon in Kenntnis gesetzt hatte und ausdrücklich betont hatte, in Zukunft sich das Imprimatur für Übersetzung vorzubehalten, waren die Reaktionen Simmels vorhersehbar; zur Sache vgl. Rammstedt 1992: $884 \mathrm{f}$. mel bei Abfassung dieser Studie um das soziologisch Gemeinsame, sondern er suchte nun das Trennende. Durkheims „De la définition des phénomènes religieux" von 1899 läßt sich als Resultat dieser Überlegungen fassen. ${ }^{7}$ Schon der Aufbau der Arbeit signalisierte dies, indem er zum einen strukturell entsprechend dem Simmel-Artikel angelegt war, zum anderen aber im Titel bereits den entscheidenden Unterschied demonstrativ betonte. Denn wenn Durkheim die Entität Religion als Summe „religiöser Phänomene" verstand, mittels derer Religion ausschließlich analysiert werden könne, so verwies das auf die methodische Vorgehensweise wie auf das inhaltliche Verständnis. Bestand Religion für Simmel - ,außer allem, was sie sonst etwa ist - in sozialen Beziehungsformen“" (GSG 5: 278), so war sie für Durkheim ,essentiellement une chose sociale“ (Durkheim 1898:25); aber soziale Form und fait social wurden nun von Durkheim nicht mehr per se als identisch angesehen, der noch in den „Regeln“ von „formes cristallisées“ angesichts Simmels „Problem der Sociologie" gesprochen hatte, denn hatte Simmel von den sozialen Formen geschrieben, daß sie in der Religion, „von ihrem empirischen Inhalt gelöst, verselbständigt und auf eigene Substanzen projizirt werden“ (GSG 5: 278f.), so sah Durkheim in Religion ein Zwangsgebilde, das in seiner Gestalt, in der es sich darstelle, ,n'est pas l'oeuvre de ces raisons, mais de l'esprit collectif". Und deutlich wird von Durkheim der Unterschied hier zu Simmel, der statt Kollektivbewußtsein Wechselwirkung angeführt hätte, hervorgehoben,wenn er dieses der Gesellschaft zuordnete: „La société a sa manière d'être qui lui est propre; donc, sa manière de penser.“(1898:25)

$* * *$

Durkheim hatte sich in seinem Beitrag vergewissern wollen, daß sein gegen Simmel aufgekommenes Ressentiment sich in einem unterschiedlichen

\footnotetext{
${ }^{7}$ Dieser Aufsatz war von Durkheim zu diesem Zeitpunkt nicht geplant gewesen. Zwar bereitet er ab Februar 1898 schon den zweiten Band der "Année sociologique" vor, zwar lag hierfür bereits der „Essai sur la nature et la fonction du sacrifice" von H.Hubert und M. Mauss vor, aber er selbst fühlte sich dann angesichts der Entwicklung der Dreyfus-Affäre verpflichtet, erst einmal auf den im März 1898 erschienenen Artikel von F. Brunetière mit "L'individualisme et les intellectuels" zu reagieren. Erst in der zweiten Jahreshälfte 1898 kann Durkheim sich dann dem religionssoziologischen Thema gewidmet haben.
} 
soziologischen Ansatz bestätigte. Als Simmel dann Durkheims „De la définition des phénomèmes religieux" las, nahm er Durkheims Distanzierung wahr, und sah im „Premier Congrès International de Philosophie" , der 1900 in Paris stattfinden sollte, den geeigneten Rahmen, um nun seinerseits auf Durkheim zu reagieren. So sagte er zu, als er im Frühjahr 1899, also kurz nach Erscheinen des Aufsatzes von Durkheim, ins „Comité de Patronage“ für den Kongreß gebeten wurde; und Simmel bot dem Kongreß an, ,eine kleine Abhandlung - das Resultat ganz neuerdings von mir geführten Untersuchungen vorzulegen. Dieselbe wird über religiöse Erkenntnistheorie (Théorie de la connaissane religieuse) handeln", wie er Xavier Léon im Sommer 1899 mitteilte(in GSG 22/23). Dieser Vortrag ${ }^{8}$ belegte zunächst, daß Simmel - wohl im Gegensatz zu Durkheim - den Diskurs aufrecht erhalten wollte, ging er doch noch von einem gemeinsamen SoziologieVerständnis aus; er fand jedoch die eigenwilligen Prononcierungen Durkheims für sich nicht akzeptabel und meinte, daB die sich darbietenden Widersprüche auf erkenntnistheoretische Mängel zurückgeführt werden könnten.

Simmel hatte immer wieder betont, daß die Erkenntnistheorie auch einer „exakten“ Soziologie vorgeordnet und die (spekulative) Sozialphilosophie ihr übergeordnet seien, so daß eine Begrenzung auf Soziologie nicht möglich sei (vgl. GSG 6: 9; GSG 11: $39 \mathrm{ff}$.; GSG 16: $84 \mathrm{ff}$.). Mit Durkheim daher Differenzen im Erkenntnistheoretischen anzunehmen, hieß Simmel, das Gespräch ins Philosophische verlagern zu müssen. Auf einem philosophischen Kongreß ein erkenntnistheoretisches Problem anzusprechen, galt als legitim und dieses in Bezug auf Religion vorzubringen, war zumindest in der deutschsprachigen Diskussion üblich, ${ }^{9}$

${ }^{8}$ Da Simmel verhindert war, wurde sein Vortrag auf dem "Congrès International de Philosophie" von Elie Halévy verlesen und verteidigt; vgl. Editorischer Bericht zu GSG 7:362. Der Vortrag erschien unter dem Titel „Beiträge zur Erkenntnistheorie der Religion" 1901 in deutsch und verspätet, 1903, in französisch unter dem Titel „De la religion au point de vue de la théorie de la connaissance" (Premier Congrès International de Philosophie. Tome II: Morale Générale, S. 319-337).

$9 \mathrm{Vgl}$. die von Volkhard Krech zusammengestellte „Bibliographie religionssoziologisch relevanter Literatur zwischen 1870 und 1918" im Rahmen des von mir geleiteten DFG-Forschungsprojektes „Religion im Spannungsfeld von Individuum und Gesellschaft: Georg Simmels Position in der religionswissenschaftlichen Diskussion um $1900^{\circ}$ ", Bielefeld Msk. 1995, S. 144-345. Siehe auch Krech 1996: S. 183-341. auch wenn Simmel als Leser z. B. der „Revue de Métaphysique et de Morale" wissen mußte, daß dies in der fanzösischsprachigen Philosophie ungewöhnlich war. Wenn Simmel also für den internationalen Philosophenkongreß einen Vortrag „'Religiöse Erkenntnistheorie' (Théorie de la connaissance religieuse)" vorlegte, so war das von Simmel wohl überlegt und als Adressat war nur Emile Durkheim ausmachbar. In dem Vortrag ging es primär um erkenntnistheoretische Fragen, die am Beispiel Religion exemplifiziert wurden; und Religion kam dabei eine doppelte Bedeutung zu, nämlich zum einen war Religion in den Aufsätzen von Simmel (1898d) und Durkheim (1899a) thematisiert worden, auf die der Diskurs fußte, zum anderen war aber Religion für Durkheim und Simmel das Paradebeispiel für „das Kollektive als das eigentlich Wirksame in der Gesellschaft".

Mochte Durkheim in seinem religionssoziologischem Text eine Definition von Religion im Rahmen einer erst in nuce faßbaren eigenen Theorie gesucht haben, so stand Simmel noch das programmatische Vorwort zum Eröffnungsheft der „Année sociologique" vor Augen, das von den Herausgebern kollektiv getragen war ${ }^{10}$ - so auch von Simmel. In der „Préface“ hatte man ein eigenes Programm aufgestellt, in dem es u.a. hieß: Die Soziologie „suppose seulement que les phénomènes so-

10 Vgl. Préface. AS 1 (1896/97), [1898]: I-VII. - Im Gegensatz zu den Préfaces der späteren Bände ist diese im Plural gehaltene Einleitung nicht mit den Initialen E.D. signiert und zudem inhaltlich offener. Als Beleg offenkundiger ist ein Brief Durkheims an Célestin Bouglé vom 26. Dezember 1897 , in dem es heißt: „Je vous envoie, par ce même courrier, comme papiers d'affaires, la Préface de l'Année sociologique. Je vous prie de la lire le plus tôt possible et de l'envoyer, aussitôt lue, à Simiand, Institut Thiers, 5 Rond Point Bugeaud, Paris. Je le préviens qu'il la recevra par votre intermédiaire. / Je vous prie de la lire au double point de vue suivant: $/ 1^{\circ}$ ) L'entende est-elle, comme je l'espère, complète sur les idées qui y sont exprimées. En cas contraire, veuillez me signaler sur quels points vous avez des scrupules. $\left(2^{\circ}\right.$ ) Dites moi tout ce qui pour vous semble utile soit à ajouter soit à retrancher, dans l'intérêt commun." (EDT 2: 416). - Auch Simmels Handschrift scheint in der „Preface" durch: schon die Bereichsaufzăhlung ist in dieser Form bei ihm häufig nachzuweisen. $\mathrm{Er}$ muß vor Bouglé, und zwar spätestens im November 1897 den Text gesehen und uberarbeitet haben, da sonst die Übereinstimmungen mit "Soziologie der Religion" nicht möglich wären. - Es ist also nicht korrekt, als Autor dieser "Préface“" nur Durkheim zu nennen, wie es tublich zu sein scheint; vgl. Viktor Karadys Bibliographie in EDT 3: 494; Lukes 1973: 563; König 1976: 404 oder K.Meier 1995: 145. 
ciaux sont liés suivant des relations intelligibles et accessibles à l'investigation scientifique. Par suite, elle n'a pas à tenir compte des systèmes qui partent de l'hypostèse contraire. Les temps sont passés où il pouvait être utile de les réfuter; si peu avancée que soit notre science, elle a dès à présent produit assez de résultats pour n'avoir pas à justifier perpétuellement ses droits à l'existence." (AS 1, 1898: V.). ${ }^{11}$ Und dieses Programm wurde als "notre programme“ vorgestellt, d. h. das Programm der Herausgeber der "Année sociologique“, also neben Emile Durkheim: Georg Simmel, Gaston Richard, Emmanuel Lévy, Célestin Bouglé, Paul Fauconnet, Henri Hubert, Paul Lapie, Marcel Mauss, Albert Milhaud, Muffang, Dominique Parodi und François Simiand. Diese Herausgeber verstanden sich als Gruppe, hieß es doch abschließend im Préface:

„Pour cette seule raison, et indépendamment des résultats utiles qu'elle peut avoir, notre tentative mérite, croyons-nous, d'être accueillie avec intérêt par tous ceux qui ont à coeur de voir la sociologie sortir de la phase philosophique et prendre enfin son rang parmi les sciences."(AS 1, 1898: VII) ${ }^{12}$

Simmel hatte stets die Auseinandersetzungen um den „wahren“ Begriff „Soziologie" als Glasperlenspielerei belächelt und seinerseits mehrmals herausgestellt, daß ,the real question is to state problems and to solve them and not at all to discuss the names which we should give to particular groups of them"(1895: 420). Die Aufgaben der Soziologie ergäben sich durch die „drängende Not der Zeit", die von ihr nur unzulänglich beachtet werde, so daß das soziologische Erkennen ,eine principielle Kritik seiner selbst“" unterzogen werden müsse (Simmel 1896:575). In diesem Sinne verstand Simmel sein Engagement, Kant für die Sozialwissenschaften $\mathrm{zu}$ rezipieren, Kant, der in Bezug auf die Naturwissenschaften „den Streit zwischen Empirismus und Metaphysik dadurch geschlichtet, daß er nachwies, wieviel Metaphysik in

"Und eingefuhrt wurde diese Feststellung mit: „Du côté de la philosophie, elle était facile à déterminer. Toutes les doctrines qui concernent les moeurs, le droit, les croyance religieuses, nous concernent pourvu qu'elles admettent le postulat qui est la condition de toute sociologie, à savoir l'existence de lois que la réflexion, méthodiquement employée, permet de découvrir. Par là, nous ne voulons pas dire qu'il faille nier toute contingence pour être sociologue; la sociologie, comme les autres sciences positives, n'a pas à se poser ce problème métaphysique.“ - Man vgl. das mit GSG 5: 52-61.

${ }^{12}$ Es ist also unrichtig, diese Personen nur als „Mitarbeiter (es gab zu keiner Zeit ein Herausgebergremium)" zu bezeichnen, wie es bei Terry N. Clark heißt (1981: 173). der Erfahrung selbst unvermeidlich enthalten ist; daß diese kein passives Hinnehmen thatsächlicher Eindrücke ist, sondern eine Bearbeitung der letzteren nach Kategorien, welche a priori in uns liegen“. Hatte Simmel in den „Problemen der Geschichtsphilosophie" demgemäß nachzuweisen versucht, „wie sehr die Geschichtsforschung auch da von apriorischen Voraussetzungen abhängig ist, wo sie rein empirisch vorzugehen glaubt, und wie viel Überempirisches in ihrer scheinbaren exakten Thatsächlichkeit steckt" (Simmel 1896: 576), so schien ihm, daß Durkheims „Définition des phénomènes religieux“ wie auch die „Préface“ zum 2. Band der "Année sociologique" nun ein Theorieverständnis vermittelten, das Empirismus gegen konstruktive Systematik setzte, eine Diskussion, die Simmel nur wieder mit Kant auf der Ebene der Erkenntnisfrage lösbar schien.

Seine „Beiträge zur Erkenntnistheorie der Religion" begann Simmel pointiert, indem er - gleichsam Durkheims Definition verunmöglichend konstatierte, daß „Religion als solche ein Vorgang im menschlichen Bewusstsein und weiter nichts ist". Erst im weiteren dann begruindete er diese Formulierung mit Rückgriff auf Kant, mit Erkenntnistheorie. Und er meinte Durkheim, wenn er anmerkte, daß Religion keine Einheit sei, ,welche seine Elemente zusammenschliesst"., daß das "religiöse Phänomen" sich nicht aus Religionsinhalten zusammensetze, ,,sondern dass es eine innere Einheit sui generis ist “, und schließlich, daß ,die religiöse Form auch an irdischen Inhalten aufzusuchen und weit jenseits der eigentlichen Religion ihre Ansätze zu finden“ seien. (GSG 7: 9,11,14)

Mochte Durkheim noch so scharf in der „Préface“ zum 2. Band der „Année sociologique" das Eigenständige seiner Soziologie der Religion umrissen haben, so zeigte doch dann Simmels erkenntnistheoretisch gestützte Kritik in Durkheims späteren Argumentationen Wirkung. Das Neue in Durkheims ,Les formes élémentaires de la vie religieuse“ von 1912 scheint ohne Berücksichtigung der Simmel-Arbeiten zur Religion nicht zu deuten. ${ }^{13}$

${ }^{13}$ Simmel veröffentlichte zwischen 1901 und 1912 elf Abhandlungen zur Religion, so auch die Monographie „Religion“, die 1906 in Martin Bubers Reihe "Gesellschaft" als 2. Band erschien. Einige dieser Texte sind Durkheim bekannt geworden. Im Gegensatz zu Simmel erfährt aber die Religionssoziologie bei Durkheim eine Änderung, die in der Monographie von 1912 faßbar wurde. - In der Sekundärliteratur wird diese Änderung durchgehend hervorgehoben, ohne daß jedoch die Linie zu Simmel gezogen wird; vgl. R.A. Jones 1981; Pickering 1984; Krech/Tyrell 1995. 
Denn Durkheims „Les formes élémentaires de la vie religieuse" brachten einleitend Überlegungen zum Untersuchungsobjekt, die im Untertitel präzisiert wurden als „Sociologie religieuse et théorie de la connaissance" (1912a: 1-28). Hatte Simmel die Erkenntnistheorie der Soziologie wie der Religionswissenschaft vorgelagert als Reflexion der menschlichen Möglichkeit, Wissenschaft zu betreiben, so fand sich das dann bei Durkheim in der Form angesprochen, daß bei ihm jene Möglichkeit als sozialen Ursprungs gedeutet wurde. Simmel hatte Kants Apriori-Lehre, die zur Lösung des Konflikts zwischen Empirismus und Metaphysik in den Naturwissenschaften gedient hatte, aufgegriffen und auf Sozialwissenschaften, die ja auch Erfahrungswissenschaften seien, übertragen; Durkheim folgte dieser Überlegung 1912, indem er als aktuelle Problemlage konstatierte, da $\beta$ erkenntnistheoretisch sich das empirische dem apriorischen Konzept unvermittelbar gegenüberständen, eine Problemlage, die Durkheim dadurch meinte meistern zu können, daß er die „,notions essentielles“, die „catégories de l'entendement“ als „dans la religion et de la religion" geboren sah, als ,produits de la pensée collective" (Durkheim 1912a: $21,12,13,14)^{14}$ ausgab. Da dieses Soziale aber nie auf ein Individuelles zurückverweisen könne, sondern auf Gesellschaft, deren Realität sui genesis sei, war Durkheims Erkenntnistheorie nicht der Soziologie vorgelagert, sondern vielmehr nur als Teil der Soziologie faßbar.

Ein weiterer, deutlicher Bezug zu Simmel fand sich dann schon im ersten Kapitel des ersten Buches („Questions préliminaires“) dieser Monographie, für die Durkheim auf seinen Aufsatz von $1899 \mathrm{zu}-$ rückgriff, den Titel ein wenig ändernd: „Definition du phénomène religieux et de la religion“; zwar unterschiede sich die jetzige von der damaligen Definition, aber es gäbe keine essentielle Änderungen in seinem Konzept, wie er gleich einleitend vermerkte(Durkheim 1912a: 31, Anm. 1). War er dort in der Definition ausschließlich vom ,caractère obligatoire" ausgegangen (Durkheim 1899a: 22, 23), um Religion nicht nur als ,fait social“ zu fassen, sondern sie zugleich letztlich mit Gesellschaft synonym zu setzen, so ging er 1912 vom Verständnis aus, daß Religion ,un système solidaire de croyances et de pratiques relatives à des choses

${ }^{14}$ Offenkundig wird der Bezug auf Simmel bei Durkheim (1912: 22), wenn er davon spricht, daß wenn ,les catégories sont des représentations essentiellement collectives, elles traduisent avant tout des états de la collectivité, also in dem, was nach Simmel soziale Formen heißt. sacrées" sei, „qui unissent en une même communauté morale, appelée Eglise, tous ceux qui y adhèrent"(Durkheim 1912a: 65). Diese Änderung wäre notwendig geworden, da sich die alte Definition als zu formal erwiesen und „trop complètement le contenu des représentations religieuses" vernachlässigt habe (Durkheim 1912a: $65 \mathrm{f}$., Anm.1). Durkheim hatte sich in seinem Aufsatz von 1899 in diese extreme Definition verstiegen, wohl auch, weil sich so der Unterschied zu Simmel fassen ließ. Dessen Verständnis der Religion als Form entsprach Durkheims ",la religion soit essentiellement une chose social" (1899a: 25); aber die bei Simmel einhergehende Frage nach dem Inhalt war bei Durkheim ausgeklammert; war die Unterscheidung in Form und Inhalt bei Simmel eine Frage der Methode, so wurde bei Durkheim die Form als „chose social" verdinglicht - und damit die Fragen nach Religionsinhalt wie nach der sozialen Funktionalität von Religion überflüssig, ${ }^{15}$ Fragen also, die sich in Zusammenhang mit dem religionsethnologischen Material im Vorfeld der „elementaren Formen des religiösen Lebens" aufdrängten.

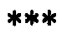

Anders als beim Thema Religion war es über Soziologie zwischen Simmel und Durkheim nicht zum Diskurs gekommen, auch wenn die Interaktion während der Gründungsphase der „Année sociologique" Spuren in den späteren soziologischen Arbeiten beider hinterlassen hatte. Simmels soziologisches Programm von 1894 fußte auf der Annahme, daß die Gesellschaft als Ursache nur Wechselwirkung heißen könne, was einerseits hie $B$, da $\beta$ er Gesellschaft mit Wechselwirkung definierte (GSG 5: 54; GSG 11: 18-21) - nicht zufällig findet sich in den frühen amerikanischen Übersetzungen seiner Arbeiten für „Wechselwirkung“ der Ausdruck ,interaction" -, zum anderen sieht er in der Wechselwirkung Ursache (vgl. GSG 2: 129138; Simmel 1896: 581; - und zur Sache KrV: A 211-215). Simmel hatte schon in Auseinandersetzung mit Dilthey (vgl. Köhnke 1989; Köhnke 1996: 380-397) auf die Gesellschaft als Ursache - ohne Gesellschaft als Einheit an sich oder als Einheit von Individuen zu reifizieren - auf Wechselwirkung verwiesen (vgl. GSG 2:131); und auch mit

15 Daß dieses Manko auch im Kreis um Durkheim gesehen wurde, darauf weist ein Brief Dominique Parodis an Célestin Bouglé aus dem Mai 1899 hin, in dem er fragt; „kommt Dir zum Beispiel seine Definition des religiösen Phănomens nicht ein bißchen materiell und außerlich vor?". (In Lepenies $2,1981: 346$ ) 
Durkheim zeichnet sich ein entsprechender Konflikt $\mathrm{ab}$, indem dieser von einem verdinglichten $\mathrm{Ge}$ sellschaftsbegriff ausging, ja, ausgehen mußte, da er die Kausalitätsfrage für seine Soziologie nicht speziell stellte (vgl. Berthelot 1989 und 1994). Durkheim mußte daher Individuum und Gesellschaft binär setzen und beide als Akteure nehmen. Wie das Individuum ist also auch die Gesellschaft ein Eigenständiges: „La société a sa manière d'être qui lui est propre; (...) Elle a ses passions, ses habitudes, ses besoins qui ne sont pas ceux des particuliers et qui marquent de leur empreinte tout ce qu'elle conçoit “" (Durkheim 1899a: 25). Mit diesem Gesellschaftsverständnis stand Durkheim daher dem Wechselwirkungsbegriff skeptisch, bzw. ablehnend gegenüber (Durkheim 1899a: 5; 1901: XVI). Wie er ihn verstand, ihn zugleich deutete, darauf weist seine Übersetzung des Begriffs „Wechselwirkung“ hin, für den er willkürlich und letztlich sinnentstellend „inter-action individuelle“ wählte (vgl. Simmel 1898b: 90 vs. GSG 5:345). ${ }^{16}$ Von diesem Verständnis von Wechselwirkung aus wird aber auch dann Durkheims Einschätzung der Simmelschen Soziologie nachvollziehbar, die er unter die Rubrik ,Sociologie psychologique" in der „Année sociologique" eingeordnet sehen wollte. ${ }^{17}$ Psychologisch schien ihm, ,toute l'idée de Simmel“, da sie bei der vorgegebenen Unterscheidung von individuell und kollektiv das Kollektive nur in den Interaktionen von Individuen annähme und die Gesellschaft damit nur als Summe der Interaktionen von Individuen verstehen könnte, also alles, auch das Kollektive als eigentliche Opposition zum Einzelwesen, nur auf individuelles Verhalten zurückführen müßte.

Durkheim lehnte bei Simmel nicht generell die psychologische Komponente ab, sondern die letztendliche Gebundenheit an die Individualpsychologie. Simmels Betonung des Kollektiven gegenüber dem Individuellen, seine Analyse sozialer Formen, die determinierenden Charakter hätten, seine von der Soziologie ausgehende Kritik der Moral - alles das war Durkheim sympathisch. Aber Simmels Selbstverständnis als Kantianer fessele ihn an dessen individualistischen Ansatz, ${ }^{18}$ hindere ihn bisher, seinen soziologischen Ansatz weiterzuverfol-

${ }^{16}$ Diese Übersetzung muß Durkheim nach Imprimierung der Übersetzung durch Simmel vorgenommen haben; denn Bouglé übersetzte Wechselwirkung mit ,action réciproque“; vgl. hierzu Simmel 1894: 499 vs. GSG 5: 54.

17 Dieser Titel wurde möglicherweise mit Zustimmung Simmels gewählt; vgl. Brief Durkheims an Bouglé vom 6. Juli 1897, EDT 2: 399.

${ }^{18} \mathrm{Zu}$ Emile Durkheims Kritik an Kant vgl. Durkheim 1898: 263, 266 und Durkheim 1950: 11. und 12. Vorlesung. gen, wie Durkheim ja mehrmals bedauernd anmerkte.

$* * *$

Es sind also nicht die paradigmatischen Unterschiede zwischen Durkheims und Simmels ,Soziologien", die die plötzliche Distanzierung Durkheims von Simmel verständlich werden ließen.

Vielmehr muß ein weiterer Aspekt angeführt werden, der mittelbar im Durkheim/Simmel-Konflikt zu spüren war. Simmel hatte 1897 in seinem Beitrag für die „Année sociologique“ allgemein auf „die Bemühungen des modernen Zionismus," hingewiesen, der die „Gruppeneinheit“ der Juden wiederherstellen wolle (GSG 5: 318), was Durkheim sofort gestrichen sehen wollen, um nicht als Zionist zu gelten und behandelt zu werden. ${ }^{19}$ Dem war Simmel wohl genausowenig nachgekommen wie der Bitte, auf die Passage über die Ehre zu verzichten. $^{20}$ In beiden Fällen änderte Durkheim dann ohne Rücksprache mit Simmel eigenmächtig dessen Text. ${ }^{21}$ Gegenüber der Toleranz, mit der

${ }^{19}$ Brief Durkheims an Bouglé vom 3. April 1898: „Es war gut, mich daran zu erinnern, daß wir schon einmal sagten, Simmel sei Jude. Aber ich bin ein wenig erstaunt darüber, daß er es mir nicht gesagt hat, als ich ihn bat, den Hinweis [in seinem] Aufsatz über den Zionismus zu streichen, da ich selbst jüdischer Herkunft sei und man mich dann als Zionisten behandeln würde" (in Lepenies 2, 1981: 310). Simmel hatte das Anliegen abgelehnt, weil er den politischen Kontext Durkheims nicht durchschaute, worauf Durkheim wahrscheinlich nicht hingewiesen hatte. $-\mathrm{Zu}$ Simmels Einstellung zum Zionismus vgl. seine Briefe an $S$ Lozinskij von 1897(?), in denen er auf die Fragen eingeht, „ob er der zionistischen Bewegung Sympathie entgegenbringe und ob er an den Erfolg von Herzls Idee glaube“; in: Böhringer/ Gründer 1976: 240-243.

20 Vgl. Brief Durkheims an Bouglé vom 16. Oktober 1897 , in dem er uber das von Simmel korrigierte und gekürzte Manuskript berichtet: „er hat es um 40 Seiten gekürzt. Ich habe noch nicht gesehen, welche Teile er gekürzt hat, und glaube jedenfalls, daß der Teil über die Ehre unverändert geblieben ist." ( in Lepenies 2, 1981: 308). Dem Teil über die "Ehre“ kam also in Durkheims Augen eine so besondere Bedeutung $\mathrm{zu}, \mathrm{da}$ er diesen Teil unmittelbar nachschlug.

21 Vgl. Brief Durkheims an Bouglé vom 25.Oktober 1897, in dem er ihm mitteilt, daß er in der Zwischenzeit das Simmel-Manuskript zur Hälfte übersetzt habe, daß insgesamt nun 40 bis 45 Druckseiten nicht überschreiten würden. "Le passage sur l'honneur a disparu." (EDT 2: 413). - Die Einlassung über die Ehre ist in der deutschen wie in der englischen Fassung erhalten geblieben; bei der von Durkheim gekürzten Passage handelt es sich um GSG 5, S. 330, Zeile 15 bis S. 333, Zeile 7 . 
theoretische Unterschiede behandelt wurden, fällt die Ungeduld und Schroffheit auf, mit der in Bezug auf „Zionismus“ und „Ehre“ Durkkheim auf Simmel reagierte - und damit bewußt die langfristig geplante Kooperation mit Simmel fraglich werden ließ. Warum?

Die soziologische Behandlung von "Ehre" stand bei Simmel keineswegs im Widerspruch zu der bei Durkheim. Die Ehre - bei Schopenhauer richtungsweisend definiert als „objektiv, die Meinung Anderer von unserm Werth, und subjektiv, unsere Furcht vor dieser Meinung" (1977: 391) - wurde von beiden als "Kohäsionsform" angesprochen, wenn auch nur von Simmel einer , ausgedehnteren Betrachtung" unterworfen. ${ }^{22}$ Dienen „die Handlungen und Unterlassungen", die die Ehre vorschreibt, immer, wie Simmel formulierte „der Selbsterhaltung bestimmter sozialer Kreise", so sei sie zugleich doch "mit einem Gefühlston ausgestattet, der sich als das Eigenste und Persönlichste des Menschen kennzeichnet, als etwas, dessen Verletzung als hauptsächlichste und schlimmste Konsequenz seine Entwertung in seinen Augen mit sich bringt" (GSG 5: 412), so führte im gleichen Sinne Durkheim Ehre in Zusammenhang mit "sentiments collectifs" an, die sich auf die Person des Individuums bezögen (1893: $88 \mathrm{f}$., 128) und hob zugleich die Bedeutung der Ehre als gesellschaftserhaltendes Element unter den Bedingungen der organischen Solidarität hervor, gerade weil die Ehre zur Individualisierung und zur Entfaltung der Persönlichkeit des Einzelnen beitrüge (Durkheim 1893: 46 f.). Aber es sind dann zwei Aspekte in dieser Diskussion, die für Durkheim große Bedeutung bekamen, nämlich die bei Simmel anklingende inhaltliche Beliebigkeit der Ehre und die ständisch hierarchische Vorstellung bei dem der Ehre zugehörigen "bestimmten sozialen Kreis“. Durkheim rechnete Ehre zu den ,kollektiven Gefühlen", die nicht vom Individualbereich inhaltlich bestimmt werden könnten; deshalb zählte Ehre für ihn zu den Phänomenen, die „à la personne de l'individu“(1893: 128) gerichtet sind, die mit die Persönlichkeit ausmachten, die immer eine soziale und nur eine soziale sei. Das lag der Auffassung

22 Bei Simmel wird Ehre häufig, in verschiedenen Zusammenhängen, angesprochen, so z. B. GSG 2: 245-246; GSG 3: 189-205; GSG 4: 377; GSG 5: 107, 330-333, 412; GSG 10: 12-14; GSG 11: 79, 396-398, 485-489, 584-585, 598605, 824-825, 827-828; GSG 14: 189, 235-236, 249. - Bei Durkheim wird „Ehre“ dem Begriff nach randständiger, der Sache nach jedoch häufig benutzt; so z. B. Durkheim 1893: 33, 43, 88, 128; Durkheim 1898: 265; Durkheim 1912b; Durkheim 1950 (dtsch. Übers.): 156, 164, 327.
Simmels jener Jahre nahe, der Persönlichkeit als „role set“, als „Kreuzung sozialer Kreise“(GSG 2: 237-257) interpretierte. Der Ehrbegriff war für ihn mit Auflösung der ständischen in der modernen Gesellschaft an soziale Rolle gebunden und damit funktional für Sondergruppierungen; das bedeute aber zugleich, daß Ehre den umfassenden Bezug auf die Lebensform verloren habe. Der Einzelne wäre je nach sozialem Kontext unterschiedlichen Ehrbegriffen unterworfen, was dazu führen könne, daß der Ehrbegriff in einem Kontext etwas forde$\mathrm{re}$, was er in andern untersage. Und als Beispiele nannte Simmel die Familien-, die Kaufmanns-, die Beamten- und die Offiziersehre (GSG 11: $600 \mathrm{f}$.).

$* * *$

Als das Thema „Ehre“ zwischen Durkheim und Simmel 1897 undiskutiert zum Streitfall wurde, wies dieses auch und vor allem für Durkheim eine politische Dimension auf. Denn 1897/98 kulminierte die Dreyfus-Affaire, die mit dem Freispruch des eigentlichen Verräters Walsin-Esterhazys (11.1. 1898) und dem Manifest Zolas „J'accuse!“ (13. 1. 1898) einen ihrer Höhepunkte fand. „In jenen Tagen des Verbrechens verzweifelte der gute Bürger am Vaterland und an der Moral Frankreichs", so umschrieb Anatole France die Situation, „Gerechtigkeit, Ehre und Vernunft, alles schien verloren" (zitiert nach Thalheimer 1963: 195). Dies mußte auch Durkheim spüren, der sich politisch betroffen fühlte, als Dreyfusard Anfeindungen ausgesetzt war (Lukes 1973: 333) und sich engagiert einsetzte. $\mathrm{lhm}$ wie vielen anderen stellte sich die in der Affäre offensichtlich werdende Front aus Royalisten, Nationalisten, Militärs und Würdenträgern der katholischen Kirche nicht nur als politische Koalition sondern als grundsätzliche Infragestellung der Dritten Republik dar. Das politische Engagement der Dreyfusard konnte sich dementsprechend nicht politisch äußern, um nicht als parteipolitisch falsch verstanden zu werden, sondern konnte sich nur gesinnungsethisch geben, um die ,agitation morale“, gespeist aus „,nos énergies social" als „un moment de la conscience humaine" (Durkheim 1904) ${ }^{23}$ erscheinen zu lassen. Vor allem die ,intellectuels“ - als Schlagwort Ergebnis der Dreyfus-Affäre - sahen es so als ihre Rolle, Stimme des Gewissens der Gesellschaft, der

${ }^{23}$ Darum hat Lukes (1973: 33, Anm. 49) recht, alle die Interpretationen scharf zu verurteilen, die Durkheims Einstellung in der Affäre als rein moralische beurteilen. 
Nation zu sein und propagierten den „Protest“ als politisch-soziale Aktion, sich nämlich in Berufung auf die eigentlichen gesellschaftlichen Werte gegen die aktuelle Regierung auszusprechen, um sie zu einer Stellungnahme zu jenen Werten zu bewegen oder zu zwingen. Durkheim griff in diese Auseinandersetzung mehrmals ein, erstmals durch seine Erwiderung auf Ferdinand Brunetières Artikel „Après le procès“, durch sein „L'individualisme et les intellectuels“. In ihm wies er darauf hin, daß der moderne Individualismus gesellschaftlich bedingt sei, gleichsam als eine säkularisierte „Religion“ angesehen werden müsse: „C'est ... le seul système de croyances qui puisse assurer l'unité morale du pays" (1898: 270).

Aber indem Durkheim die Formulierung wählte: „Quiconque attente à une vie d'homme, à la liberté d'un homme, à l'honneur d'un homme“" (1898: 265; vgl. Durkheim 1893: 128), also Leben, Freiheit, Ehre in eine Reihe stellte, zeigte dieses doch einen anderen Stellenwert von Ehre auf, als bei Simmel zu finden war. Das Durkheim'sche Verständnis korrespondierte mit dem von Dreyfus und den Dreyfusards; wenn Hauptmann Dreyfus während der Verhandlungen des Kriegsgerichts 1894 notierte: „Wenn ich diesen entsetzlichen Kampf überstehe, in dem man mir meine Ehre nehmen will, so halte ich ihn nur aus, weil ich die Ehre meines Namens... verteidigen will. (...) Ich habe für meine Ehre gelebt"(nach Thalheimer 1963: 58), wenn Emile Zola in seiner Anklage den Präsidenten an seine Ehre erinnerte, oder das Reden von der „Ehre der Armee“ (nach Thalheimer 1963: 185, 191) entlarvte, immer klang hier Ehre als Aspekt einer vitalen, individuellen Identität an. Schon Hauptmann Dreyfus meinte, wenn er von seiner Ehre sprach, nicht mehr nur die Ehre des Offizier-Kreises, sondern er meinte eine generelle Ehre des Gesellschaft-Kreises, die Grundlage sei, sich selbst noch achten zu können. Ehre war weitgehend hier identisch mit dignitas, mit Menschenwürde (Dreyfus 1991; zur Problematik: Weinrich 1971, Zingerle 1991).

Und entsprechend fand sich Ehre während der Dreyfus-Affäre bei Durkheim angesprochen. Mochte er die Infragestellung seiner Rollen als Hochschullehrer, als Intellektueller, als (politischer) Bürger anfangs noch energisch entgegentreten - Ehre in diesem Zusammenhang noch als Sozialprestige faßbar -, so war ein adäquates Reagieren nicht mehr möglich, als ihm unlauteres Handeln nachgesagt wurde, das seine soziale Rolle und darüber hinaus seine Persönlichkeit obsolet erscheinen lassen sollten. Sein Ansehen schien ihm gefährdet. Und das weitete sich zur existenziellen Bedrohung, als er den mit der Dreyfus-Affäre einhergehenden Antisemitismus zu spüren bekam. ${ }^{24}$ Wie Dreyfus war ja auch Durkheim Jude, wie er stammte er aus einem französisch-deutschen Grenzgebiet, jener aus dem Elsaß, dieser aus Lothringen, wie er hatte er einen deutsch klingenden Namen - alles das machte ihn von vornherein verdächtig. Und in Vorwegnahme des möglichen, bzw. latent immer erhobenen Vorwurfs, er sei als Jude per se Internationalist oder gar Zionist und als Lothringer mit deutschem Namen per se germanophil und stets bereit, Frankreich zu verraten, versuchte Durkheim, jede Verdachtsverstärkung zu verhindern. Denn war der Verdacht einmal artikuliert, dann war man ja in jenem Teufelskreis, aus dem man, wie der Fall Dreyfus zeigte, nicht aus eigener Kraft herauskam.

Trotz der politischen Hoffnungen, die man ab 1897 in den Reihen der Dreyfusard ausmachen konnte, eskalierten die antisemitischen Ausschreitungen: in den Straßen wurden jüdische Geschäfte demoliert, in sog. patriotischen Veranstaltungen ,Tod den Juden!"“ skandiert, in den Universitäten jüdische Professoren von Studenten niedergebrüllt; 1898 wurde ,zum traurigsten Jahr in der neuesten Geschichte der französischen Juden“ und im gleichen Jahr erreichte ,die Progromwelle in Algerien ihren Höhepunkt" (Dubnow 1929: 238, 239). Durkheim wußte von diesen Ereignissen, hatte sie in Bordeaux selbst miterleben müssen. Nur vor diesem Hintergrund wird verständlich, weshalb er in seiner Erwiderung auf Brunetière, ,one of the immortals of the strongly anti-Dreyfusard Académie Française" (Lukes 1973: 335), nicht auf dessen ersten Teil einging, nämlich auf die Frage nach den Gründen für den Antisemitismus, für den gerade die gebildeten Juden z.T. selbst verantwortlich seien, auch nicht den zweiten Teil, in dem die Rolle der Armee in der Demokratie erörtert wurde, anspricht, sondern nur den dritten Teil mit der enthusiastischen Auseinandersetzung mit den Intellektuellen. Als ein solcher konnte er Brunetiére als Kollegen entgegentreten und wie selbstverständlich immer wieder Gemeinsames anklingen lassen, seien es Wertmaßstäbe, politische Fundamental-

${ }^{24} \mathrm{DaB}$ sich der Antisemitismus in Frankreich schon früher schnell ausbreiten konnte, dafür macht Dubnow (929: 226-248) eine Reihe von sozialen, ökonomischen und politischen Gründe verantwortlich. Die sich Anfang der $80 \mathrm{er}$ Jahre kristallisierende antisemitische Front entsprach schon der dann in der Dreyfus-Affäre; vgl. auch Raphael 1992. 
ansichten oder moralische Regeln. Aber über Antisemitismus ließ sich zwischen dem Juden Durkheim und dem Nicht-Juden Brunetière so nicht sprechen, denn durch die im Antisemitismus unterstellten Ausgrenzungen war ein politisches, soziales, moralisches Gemeinsames nicht mehr beschwörbar.

Wenn Durkheim also hier auf die Antisemitismusfrage nicht einging, so geschah das gezielt, gerade auch weil er sich durch den Brunetière-Aufsatz betroffen fühlte. ${ }^{25}$ Und 1899 ist jener angesprochene Hintergrund dafür auch entscheidend, wie er die Frage nach dem Antisemitismus beantwortet, denn als oberstes Ziel nennt er: „réprimer sévèrement toute exitation à la haine de citoyens les uns contre les autres“ (Durkheim 1899b:254). Nicht auf die Antisemitismus-Argumentation Brunetières eingegangen zu sein, gab sich also dem Vorsatz verpflichtet, die bürgerkriegsverdächtige Situation in Frankreich während der Dreyfus-Affäre nicht zu verschärfen; und dies sei zu erwarten, wenn man die Antisemitismus-Diskussion anheize, wenn er in ihr eine scharfe eigene Position bezöge. Aber dieser Vorsatz prägte zugleich auch diese Äußerung Durkheims zu ,Antisemitismus und soziale Krise“ von 1899 selbst. Denn, ,il y aurait lieu, je crois, de distinguer entre l'antisémitisme français et l'antisémitisme étranger qui me paraissent être deux phénomèmes de signification très diffé-

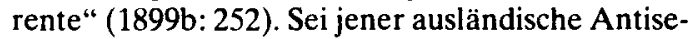
mitismus, "l'antisémitisme allemand ou russe" chronisch und traditionell, so der französische durch soziale Krisen bedingt und daher vorübergehend; sei jener aristokratischen Charakters, „il est fait de dédain et de morgue", so sei dieser durch gewalttätige Leidenschaften inspiriert, „qui cherchent à s'asseoir par tous les moyens"(Durkheim 1899b: 252), sei jener den aristokratischen politisch-sozialen Systemen immanent, so werde dieser im demokratischen System von der irrational gelenkten, destruktiven Masse als Ventil in einem „état de malaise social" (Durkheim 1899b: 253) aufgegriffen.

Indem Durkheim hier glauben machen wollte, daß es einen grundsätzlichen Unterschied zwischen dem französischen und dem deutschen Antisemitismus gäbe, negierte er von vornherein die Internationalität des Phänomens - zumindest für Fran-

${ }^{25} \mathrm{Vgl}$. Brief Durkheims an Bouglé vom 18. März 1898: „Moi aussi, javais songé à écrire quelque article, sociologique sur la questions et le plat article de Brunetière me donne la démangeaison de réprondre. Malheureusement, le temps me manque"(EDT 2: 418). kreich $^{26}$ - und steigerte zugleich den Gegensatz Frankreich/Deutschland.

Durkheim war durch die antisemitischen Ausschreitungen zutiefst betroffen, erinnerte er sich doch auch an entsprechende Aktionen in Lothringen, als Franzosen gegen Juden im Zusammenhang mit dem deutsch-französischen Krieg vorgingen - , j'ai pu, alors, l'observer de près. C'est aux juifs qu'on s'en prenait pour des defaites" (Durkheim 1899b: 252) -; aber nach außen spielte er das Thema herunter. Das Thema Antisemitismus aus der öffentlichen Diskussion herauszunehmen, schien ihm politisch opportun; die Ursachen des Antisemitismus lägen ja auch nicht im Religiösen oder Ethnischem sondern in sozialen, ökonomischen Krisen.

Durkheim stimmte eigentlich mit Simmels distanzierter Haltung zum Zionismus überein, sah wie dieser in der Assimilation die Lösung, ${ }^{27}$ trotzdem ist eine Reaktion Durkheims auf Simmels Erwähnung des Zionismus in seinem Beitrag für ,l'Année sociologique" $z u$ konstatieren, die unangemessen wirken mußte. Er wollte zuerst wohl den Begriff „Zionismus“ in seiner Zeitschrift unmittelbar nach dem 1. Zionistischen Weltkongreß, der im August 1897 in Basel stattgefunden hatte und fast durchgängig die Fronten in den Auseinandersetzungen uber den Antisemitismus verhärtet hatte, nicht erwähnt wissen, um damit alles zu vermeiden, was den einen Franzosen gegen den anderen aufbringen könnte. Simmel gegenüber aber hatte Durkheim seine Bitte um Streichen des Hinweises auf Zionismus damit begründet, ,da ich selbst jüdischer Herkunft sei und man mich dann als Zionisten behandeln würde". ${ }^{28}$ D.h., er ging wie selbstverständlich davon aus, daß ein Hinweis auf sein Jude-Sein ausreiche,um die Unvereinbarkeit von ihm als Herausgeber der Zeitschrift und der Nennung von "Zionismus" einzusehen. Simmels ihm harmlos klingende Formulierung zum „Bemühen des modernen Zionismus, ihre (sc. der Juden) Gruppeneinheit umfassend wiederherzustellen, knüpft daran an, sie wieder lokal zusammenzusiedeln“ (GSG 5: 318), ${ }^{29}$ bekam ihre politische aktu-

26 Die gegenteilige Auffassung vertrat Theodor Herzl, siehe: "The Dreyfus Affair" und "Conditions in France" in Lewisohn 1955: 194-207, bzw. 208-211.

27 „D'ailleurs les juifs perdent leurs caractères ethniques avec une extrême rapidité. Encore deux générations et c'état chose faite"(Durkheim 1899b: 253).

28 Brief Emile Durkheims an Célestin Bouglé vom 3. April 1898 (Lepenies 2 1981: 310).

29 Ausdrucklich soll noch einmal darauf hingewiesen werden, daß dieser Text von Simmel vor August $1897 \mathrm{ge}$ schrieben wurde, also vor dem Basler KongreB. 
elle Bedeutung durch die Einbeziehung der im „Baseler Programm" fixierten Forderungen der Zionisten nach Schaffung des ,Judenstaates“, das zum einen von der Unhaltbarkeit der Lage der Juden in der Diaspora und von der Einheit aller Juden ausging.

In Frankreich fand der Kongre $B$ und die auf inn erhobenen Forderungen große Beachtung, die verstärkt wurden durch den Umstand, daß als lingua franca auf dem Weltkongre $\beta$ deutsch diente und zudem der Dreyfus-Prozeß als „Auslöser" des aktuellen Zionismus häufig genannt worden war, ${ }^{30}$ Der 1.Zionistische Weltkongreß war gleichsam ÖI auf die flammenden Auseinandersetzungen um den Juden Dreyfus in Frankreich. Wurde drohend einerseits daran erinnert, daß schon einmal die Vertreibung aller Juden aus Frankreich Inhalt einer Gesetzesinitiative gewesen war, ${ }^{31}$ wurde in öffentlichen Versammlungen der Ausschluß der Juden aus dem Staatsdienst gefordert, was auf die Aufhebung der jüdischen Gleichberechtigung zielte (vgl. Dubnow 1929: 234), so meinten sich viele französische Juden durch gerade diesen Kongreß verraten, waren sie doch auf ihm als "neue Marranen" abgestempelt worden, ${ }^{32}$ war den Assimilier-

30) Theodor Herzl bekannte in einer Rede: „Zum Zionisten hat mich der Dreyfus-Prozeß gemacht, dessen Zeuge ich in Paris 1894 war. ... Und auch der Wutschrei der Menge auf der Straße vor der Ecole Militaire gellt mir noch unvergeßlich in den Ohren: 'A mort, à mort les juifs!' Tod allen Juden, weil dieser eine ein Verräter war! ... Aber der Fall Dreyfus enthält mehr als einen Justizirrtum; er enthält den Wunsch der ungeheuren Mehrheit in Frankreich, einen Juden und in diesem einen alle Juden zu verdammen. Tod den Juden! heult die Menge, als man dem Hauptmann seine Tressen vom Waffenrocke riB. Und seither ist das 'Nieder mit den Juden!' ein Feldgeschrei geworden. Wo? In Frankreich! Im republikanischen, modernen, zivilisierten Frankreich, hundert Jahre nach der Erklärung der Menschenrechte." Zitiert nach Dubnow 1929: $313 \mathrm{f}$.

${ }^{31}$ Der Vorschlag kam 1891 in der französischen Abgeordnetenkammer zur Sprache, wurde aber bereits im Vorfeld abgelehnt. Vgl. Dubnow 1929: 231.

32 In der eindrucksvollen Rede, die auf diesem gehalten wurde, hatte Max Nordau das „Gesamtbild von der Verfassung der Judenheit“" entworfen, in der die Emanzipation der Juden als Schein entlarvt wurde; „Der emanzipierte Jude ist haltlos“, führte Nordau aus, ,unsicher in seinen Beziehungen zu den Nebenmenschen, miBtrauisch gegen die geheimen Gefühle selbst der Freunde. Seine besten Kräfte verbraucht er in der Unterdrückung und Ausrottung oder mindestens in der mühsamen Verhüllung seines eigensten Wesen, denn er besorgt, daB dieses Wesen als judisch erkannt werden möchte, und er hat nie das Lustgefuhl, sich ganz zu geben, wie er ist, er selbst zu sein...". Zitiert nach Dubnow 1929: 324. ten als den Scheinapostaten der Neuzeit doch nachgesagt worden, daß sie innerlich völlig ausgehöhlt, ein Symptom des ,seelisch kranken Judentums“ seien. Durkheim wollte unter diesen Umständen den Begriff "Zionismus" nicht in seiner "l'Année sociologique" erwähnt wissen - er verwendet ihn auch in "l'Antisémitisme et crise sociale" nicht, spricht da nur von ,les aspirations vaguement religieuses" -, ja, er sah wohl in dem von Simmel wertfrei verstandenen Hinweis auf den $\mathrm{Zi}$ onismus zur Illustration der These von der Bedeutung des Raumes für die Gemeinschaftsbildung unmittelbar ein Politicum, müßte doch jeder in dieser Erwähnung des Zionismus einen Hinweis auf den geforderten Judenstaat sehen, der fatal korrespondiere mit der Antisemitismusforderung, die Juden aus Frankreich zu vertreiben.

Wie im Falle der "Ehre" ist es auch in dem des "Zionismus" keine theoretische Unvereinbarkeit, die Durkheim zum willkürlichen Eingriff in das Simmel-Manuskript verleiteten, sondern es waren wohl die politischen Umstände während der Dreyfus-Affäre, die Durkheims Handeln prägten. Er sah sich antisemitischen Anfeindungen ausgesetzt, die er in ähnlicher Form schon in seiner Kindheit erlitten hatte, und er wollte allem vorbeugen, was antisemitische Aktionen schüren konnte. So wollte er den Antisemitismus nicht auf die Ebene des Diskurses verlagert wissen und vermied und verhinderte alles, was dahin zu tendieren schien. Und er wollte nicht in die Lage versetzt werden, als germanophil zu gelten, was wegen seiner Herkunft aus Lothringen und seines Namens nahe lag, aber während der Dreyfus-Affäre sich ruinös auswirken konnte, war doch Deutschfreundlichkeit mit dem Verdacht des Vaterlandsverrats gekoppelt und Jude mit Internationalismus und Aufkündigung der Staatsbürgerschaft. Brisant mußte daher für Durkheim das Eröffnungsheft der „Année sociologique" durch die politischen Umstände kurzfristig geworden sein, denn es schien schon gewagt, $\mathrm{da} \beta$ ein Franzose und ein Deutscher für die beiden zentralen Artikel verantwortlich zeichneten, so wohl erst recht, wenn zudem beide Juden waren. Und wenn dann der deutsche Jude wie selbstverständlich von Zionismus redete, so ließ sich davon ausgehen, da $B$ das mit Billigung des französischen Juden geschehen war. 1898 in Frankreich Zionismus als Selbstverständlichkeit hinzustellen, war schon unerhört, dieses aber durch einen deutschen Juden geschehen zu lassen, das war ein politischer Affront, bewußt durch den französischen Juden lanciert. So mußte wohl Durkheim gedacht haben, denn er schrieb ja ausdrücklich, daß eine Erwäh- 
nung von „Zionismus“ in Simmels Beitrag dazu führe, daß "man mich dann als Zionisten behandeln würde". ${ }^{33}$

\section{****}

Als 1899 der zweite Band der „Année sociologique" erschien, fanden sich die Namen von Milhaud und Simmel nicht mehr unter den Herausgebern. Die wohl langfristig geplant gewesene Kooperation zwischen Durkheim und Simmel ${ }^{34}$ hatte also ein schnelles Ende gefunden; aber dafür war primär die politische Konstellation des Jahres 1898 verantwortlich zu machen - nicht jedoch wissenschaftliche Differenzen. Daß Durkheim in dieser Situation alles unterlassen sehen wollte, was die Antisemitismus-Diskussion verschärfen könnte, $\mathrm{da} B$ er Angst hatte, als französischer Jude und als lothringischer Franzose ausgegrenzt zu werden, und daß er daher jedem Verdacht vorbeugte, er sei eigentlich germanophil oder internationalistisch. wird verständlich; und dieser Druck steuerte zusehend sein Verhalten dieser Monate und Jahre. Darüber zu sprechen und dieses zu rechtfertigen, unterblieb wohl, zumindest gegenüber Simmel. $\mathrm{Da} \beta$ sich Durkheim brüsk von Simmel abwandte, mußte von diesem rein persönlich genommen werden, wußte er doch nichts von der Situation Durkheims und schätzte auf Distanz die Dreyfus-Affäre eher als ein kulturpolitisches Ereignis ein. ${ }^{35} \mathrm{Er}$ fühlte sich durch Durkheim düpiert, hielt aber erst noch an der gemeinsamen Idee "Soziologie“ fest. Diesés beabsichtigte aber nicht Durkheim. Hatte dieser noch in seinem Beitrag zur „Revue de Métaphysique et de Morale“, nämlich in „Représentation individuelles et représentations collectives" 1898 die Chance gesucht, seinen soziologischen Ansatz separiert von dem Simmels faßbar werden zu lassen, so reagierte darauf Simmel mit „Il problema della sociologia“, das 1899 in "La riforma sociale" erschien. Hatte sich Durkheim zwischenzeitlich im zweiten Band der ,Année sociologique“ (1899) von Simmels „Zur Soziologie der Religion" (1898) distanziert, so tat er es jetzt auch von dessen soziologischem Ansatz, indem er seinen

${ }^{33}$ Brief Durkheims an Bouglé vom 3. April 1898 (Lepenies 2, 1981: 310.

${ }_{34}$ Vgl. Brief Durkheims an C. Bouglé vom Dezember 1896 (EDT 2: 394).

35 In einem von Klaus Christian Köhnke gefundenen, möglicherweise von Simmel geschriebenen Zeitungsbeitrag vom Juli 1899, wird unter dem Titel „Der deutsche Student und die ethische Bewegung" in diesem Sinne die „Dreyfus-Angelegenheit" behandelt (ev. in: GSG 17).
Bericht für die „Rivista italiana di sociologia“ von 1900 über „La sociologia e il suo dominio scientifico" nutzte, um scharf, ja teilweise polemisch mit Simmels Soziologie abzurechnen, eine Kritik, die er dann noch einmal in der mit Paul Fauconnet gemeinsam verfaßten Studie zu ,Sociologie et sciences sociales" von 1903 aufgreift. Sieht man aber einerseits die dementsprechend scharfen, bis ins Unsachliche abgleitenden Rezensionen, die Durkheim 1902 zu Simmels „Philosophie des Geldes“ (AS 5: 140-145), 1904 zu „Über räumliche Projektionen socialer Formen" (AS 7: 646f.) und zur amerikanischen Übersetzung von „Die quantitative Bestimmtheit der Gruppe"(AS 7: 647-649) geschrieben hat, so fällt demgegenuber ein anderes Verhalten Durkheims auf, wenn er sich gegen das Argument zur Wehr setzte, er knüpfe in seinen sozialwissenschaftlichen, moralwissenschaftlichen bzw. religionswissenschaftlichen Argumentationen an eine deutsche Diskussion an. Und hierbei wurde ihm zumeist Simmel als Bezugsperson vor Augen geführt.

Diese von Deploige initiierte Affäre $^{36}$ bestärkte die politisch bedingte Frontstellung Durkheims gegenüber Simmel. Bis Anfang des Ersten Weltkrieges betonte Durkheim in der Offentlichkeit diese Art der Distanz, was ihn aber wohl nicht hinderte, privat mit Simmel freundschaftlich zu verkehren und sich von Zeit zu Zeit zu treffen. ${ }^{37}$

\section{Literatur}

\section{Abkürzungen}

AS = L'Année sociologique

EDT = Emile Durkheim: textes, 3 Bde. $\mathrm{Hg}$. von V. Karady. Paris: Minuit 1975

GSG = Georg Simmel-Gesamtausgabe. Hg. von O. Rammstedt. Frankfurt a.M.: Suhrkamp 1989 ff.

$\mathrm{KrV}=$ Immanuel Kant: Kritik der reinen Vernunft. 2. Aufl., Akademie-Ausgabe, Bd. III

Berthelot, J.-M., 1989: Principe de causalité et raisonnement expérimental chez Durkheim. Revue philosophique 1: $25-50$

Berthelot, J.-M., 1994: Le status de la causalité chez Durkheim et chez Weber, in Hirschhorn, M./Coenen-

36 Vgl. Deploige 1911; in der 2. Aufl. (1923) findet sich der Konflikt mit Durkheim von 1907 und 1913 dokumentiert (S. 352-378). - Vgl. auch Firsching 1995.

${ }^{37}$ Simmel sagte z. B. 1904 seine Teilnahme am KongreB in St. Louis anläBlich der Weltausstellung ab, um sich mit Durkheim und Tarde in Frankreich treffen zu können; vgl. Levine 1993: 78 . 
Huther, J. (Hrsg.), Durkheim et Weber: Vers la fin des malentendus? Paris: L'Harmattan, S. 135-147

Besnard, Ph., 1979: La formation de l'équipe de l'Année sociologique. Revue française de sociologie 20:7-31

Böhringer, H./Gründer, K. (Hrsg.) 1976: Ästhetik und Soziologie um die Jahrhundertwende: Georg Simmel. Frankfurt a.M.: Klostermann

Bouglé, C., 1895: Les sciences sociales en Allemagne, les méthodes actuelles. Paris: Alcan , 3. Aufl. 1912

Bouglé, C., 1898: Rezension: P. Barth: Die Philosophie der Geschichte als Sociologie. Leipzig 1897. AS 1: 116123

Clark, T. N., 1981: Die Durkheim-Schule und die Universităt, in Lepenies 2, 1981: 157-205

Deploige, S., 1923: Le conflit de la morale et de la sociologie [1911], 3. Aufl. Paris: Nouvelle Librairie nationale

Dreyfus, A., 1991: Lettres du capitaine, in Gautheron, M., Hrsg.: L'honneur: Image de soi ou don de soi un idéal équivoque. Editions Autrement-Série Morales Nr.3, Mars, S. 87-88

Dubnow, S., 1929: Weltgeschichte des jüdischen Volkes. Bd. X: Das Zeitalter der zweiten Reaktion (18801914). Berlin: Jüdischer Verlag

Durkheim, E., 1893: De la division du travail social, 9.Aufl. Paris: PUF 1973

Durkheim, E., 1894: Les règles de la méthode sociologique. Paris: PUF 1983

Durkheim, E. 1898: L'individualisme et les intellectuels, in Durkheim 1987: 261-278

Durkheim, E., 1899a: De la définition des phénomènes religieux. AS II (1897/98): 1-29

Durkheim, E., 1899b: [Antisémitisme et crise sociale], in EDT 2: 252-254

Durkheim. E., 1901: Préface de la seconde edition. In: Règles de la méthode sociologique. 21. Aufl. Paris 1983

Durkheim, E., 1904: L'élite intellectuelle et la démocratie, in Durkheim 1987: 279-281.

Durkheim, E., 1912a: Les formes élémentaires de la vie religieuse: le systéme totémique en Australie, 4. Aufl. Paris: PUF 1960

Durkheim, E.,1912b: Remarque sur le problème de l'honneur, EDT 2: 385-386;

Durkheim, E., 1950: Leçons de sociologie: Physique des moeurs et du droit, hrsg. von $H$. N. Kubali und $G$. Davy, Paris: PUF

Durkheim, E., 1987: La science sociale et l'action, hrsg. von J.-C.Filloux, 2. Aufl. Paris: PUF

Firsching, H., 1995: Emile Durkheims Religionssoziologie - made in Germany? Zu einer These von Simon Deploige, in Krech/ Tyrell, Hrsg., 1995: 351-363.

Jones, R.A., 1981: Robertson Smith, Durkheim, and sacrifice. Journal of the History of the Behavioral Sciences 17: 596-627

König, R., 1976: Durkheim-Bibliographie in Käsler, D., Hrsg., Klassiker des soziologischen Denkens, Bd. I. München: C.H.Beck: $401-444$

Köhnke, K.Ch., 1989: Die Wechselwirkung zwischen Diltheys Soziologiekritik und Simmels soziologischer Methodik. Dilthey-Jahrbuch 6: 303-326
Köhnke, K. Ch., 1996: Der junge Simmel in Theoriebeziehungen und sozialen Bewegungen. Frankfurt a.M.: Suhrkamp

Krech, V. / Tyrell, H. (Hrsg.),1995, Religionssoziologie um 1900. Würzburg: Ergon

Krech, V./ Tyrell, H., 1995: Religionssoziologie um die Jahrhundertwende, in Krech/Tyrell, Hrsg: 11-78.

Krech, V., 1996: Religion und Leben. Studien zu Georg Simmels Religionstheorie. Diss. Bielefeld

Lepenies, W. (Hrsg.), 1981: Geschichte der Soziologie. 4 Bde. Frankfurt a.M.: Suhrkamp

Levine, D.N., 1993: Howard Woodhead - An American Correspondent on Simmel. Simmel Newsletter 3: 74 78.

Lewisohn, L. (Hrsg.), 1955: Theodor Herzl: A Portrait for this Age. Cleveland und New York: The World Publishing Company

Lukes, St., 1973: Emile Durkheim. His Life and Work:A Historical and Critical Study. Harmondsworth: Penguin 1975

Meier, K., 1995: Gibt es einen „Bruch“ in Durkheims früher Religionssoziologie?, in Krech/ Tyrell, Hrsg.: 129159

Pickering, W.S.E., 1984: Durkheim's Sociology of Religion. London

Rammstedt, O., 1992: Editorischer Bericht in GSG 11: 877-906

Raphael, F., 1992: Les juifs de France, de l'affaire Dreyfus au bicentenaire de la Révolution: La synthèse „francojudaïque" et sa fragilité, in Le Goff, J./ Rémond, R., Hrsg.: Histoire de la France religieuse, tom. IV: Société sécularisée et renouveaux religieux. Paris: Seuil: 253335.

Schopenhauer, A., 1977: Parerga und Paralipomena I, 2. Teilband: Aphorismen zur Lebensweisheit. Zürcher Ausgabe, Bd. 8, Zürich: Diogenes

Simmel, G., 1893: Einleitung in die Moralwissenschaft. Bd. II. GSG 4

Simmel, G., 1894a: Das Problem der Sociologie. GSG 5: $52-62$

Simmel, G., 1894b: Le problème de la sociologie, übers. von C. Bouglé. Revue de Métaphysique et de Morale 2: 497-504

Simmel, G., 1895: Supplementary Note zur amerikanischen Übersetzung des „Problems der Sociologie“: Annals of the American Academy of Political and Social Science 6: 412-423

Simmel, G., 1896: Zur Methodik der Socialwissenschaft. Jahrbuch für Gesetzgebung, Verwaltung und Volkswirtschaft im Deutschen Reich 20: 575-585

Simmel, G., 1898a: Ankündigung von l'année sociologique, vol I. Jahrbuch für Gesetzgebung, Verwaltung und Volkswirtschaft im Deutschen Reich 22: $1044 \mathrm{f}$.

Simmel, G., 1898b: Comment les formes sociales se maintiennent. AS 1: 71-109

Simmel, G., 1898c: Die Selbsterhaltung der socialen Gruppe. Sociologische Studie. GSG 5: 311-373

Simmel, G., 1898d: Zur Soziologie der Religion. GSG 5: $266-287$ 
Simmel, G., 1899a: Zur Psychologie und Soziologie der Lüge. GSG 5: 406-420

Simmel,G., 1899b: Ueber Geiz, Verschwendung und Armut. GSG 5: 529-543

Simmel, G.,1901: Beiträge zur Erkenntnistheorie der Religion. GSG 7: 9-21

Simmel, G., 1903: De la religion au point de vue de la théorie de la connaissance. GSG 19

Simmel, G., 1906: Die Religion. GSG 10: 39-119

Simmel, G., 1908: Soziologie. Untersuchungen über die Formen der Vergesellschaftung. GSG 11
Simmel, G., 1917: Grundfragen der Soziologie (Individuum und Gesellschaft) GSG 16: 59-151

Thalheimer, S.(Hrsg.) 1963: Die Affäre Dreyfus. München $\mathrm{dtv}$

Weinrich. H., 1971: Mythologie der Ehre, in Fuhrmann, M., Hrsg.: Terror und Spiel. München: Fink, S. 341-356

Zingerle. A., 1991: Historische und systematische Voruberlegungen zu einer Soziologie der Ehre. Soziologisches Jahrbuch/Annali di Sociologia 7 (II): 15-32 